\title{
Optical frequency shifter on SOI using thermo-optic serrodyne modulation
}

\author{
Yanlu Li, Stijn Meersman, and Roel Baets \\ Photonics Research Group \\ INTEC Department \\ Ghent-University - IMEC \\ 9000 Ghent, Belgium \\ Email: Yanlu.Li@intec.ugent.be
}

\begin{abstract}
An optical frequency shifter on SOI using thermooptic serrodyne modulation is fabricated. It creates a $1 \mathrm{kHz}$ frequency shift in $1550 \mathrm{~nm}$ laser, with a second-order harmonic sideband suppression of $\mathbf{- 3 9} \mathrm{dB}$.
\end{abstract}

\section{INTRODUCTION}

Laser Doppler velocimetry (LDV) is a non-contact velocity measurement technology, and it has been used in variety of fields, such as the aerospace [1], acoustics [2], and medicine [3]. Most LDV setups employ the heterodyne interferometer technique, whereby optical frequency shifters (OFSs) are required. OFSs are used to provide LDVs with the ability to sense the velocity directions [4]. It also shifts the working band away from the noisy low frequency, and greatly enhanced the signal to noise ratio (SNR) [5]. In the heterodyne interferometer, as shown in figure 1 , a coherent radiation from a laser source is split into two signals in the optical splitter: a reference signal which is frequency shifted by $f_{L O}$ in the OFS, and a measurement signal which is sent out to the moving object. The measurement signal is back-scattered by the moving object, and frequency shifted because of Doppler effect. A portion of the measurement signal is coupled back into the interferometer, and mixed with the reference light in the optical combiner. The mixed light signal is received by a photodetector. Since the frequencies between the measurement and reference lights are different before combining, an optical intensity beat is generated at the combiner. The time-variant optical beat frequency $f_{B}(t)$ is detected in the photodetector, and reveals the instantaneous axial velocities of the moving object $v(t)$ with a linear relation

$$
f_{B}(t) \approx f_{L O}-\frac{2 f_{0}}{c} v(t),
$$

where $f_{0}$ is optical frequency of the laser, and $c$ is the speed of light in the medium surrounding the moving object. Bragg cells are the most commonly used OFSs in bulk heterodyne LDVs.

Miniaturized heterodyne LDVs have been investigated by many groups. As compared to bulk LDVs, miniaturized photonic devices can provide advantages such as low power consumption and extremely compact size. However, little work has been done on silicon substrate. One important reason is that the unstressed monocrystalline silicon shows neither a

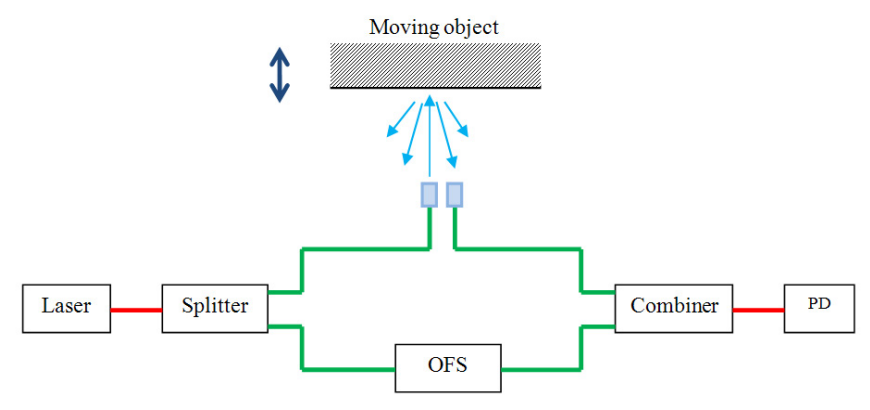

Figure 1. Schematic of the heterodyne interferometer.

strong electro-optic effect nor a strong piezoelectric effect, and those effects are commonly used mechanisms for phase modulation in the OFS in other materials [6]. Actually, many different types of phase modulations have been reported for silicon photonics in recent years, such as the thermo-optic modulation [7], carrier injection and depletion [8], and the surface acoustic wave (SAW) modulation [9]. Considering the advantages of silicon photonics [10], it is interesting to realize an OFS on silicon chip.

In this paper we demonstrate a serrodyne optical frequency shifter on silicon on insulator (SOI) using the simple thermooptic effect. The theory of serrodyne modulation is discussed in the following section. In the third section, experimental results are discussed. The conclusion is found in the last part of this paper.

\section{SERRODYNE MODULATION}

Serrodyne modulation can be used to generate optical frequency shifts and has been reported previously [6]. An optical signal $A_{0} e^{i 2 \pi f_{0} t}$ ( $A_{0}$ is the amplitude of the electric field) is phase modulated and the output can be expressed as $A_{0} e^{i\left(2 \pi f_{0} t+\phi(t)\right)}$. If the phase is linearly increasing in the time domain, i.e. $\phi(t)=2 \pi \alpha t$, the frequency of the output is actually shifted by $\alpha$ since the output is $A_{0} e^{i 2 \pi\left(f_{0}+\alpha\right) t}$. This modulation format is also called single sideband suppressed carrier (SSB-SC) modulation. However, linearly increasing the phase for a very long time is not implementable. An alternative method is to modulate the phase in a sawtooth shape, with the 


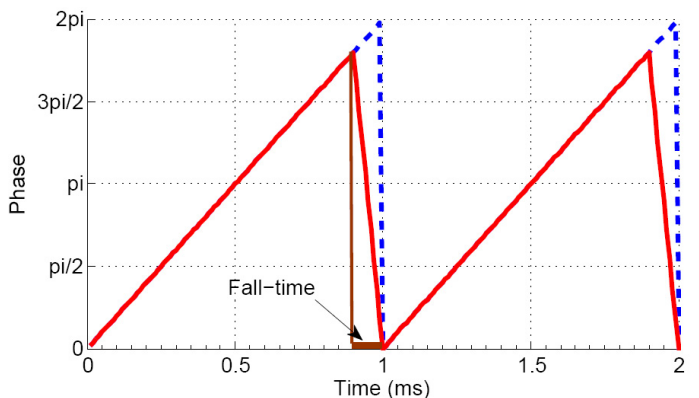

Figure 2. Ideal sawtooth phase shift (dotted blue curve) and non-ideal sawtooth with a finite fall-time $T_{F}=0.1 \mathrm{~ms}$ (red solid curve). The repetition rate of the sawtooth is $f_{s t}=1 \mathrm{kHz}$.

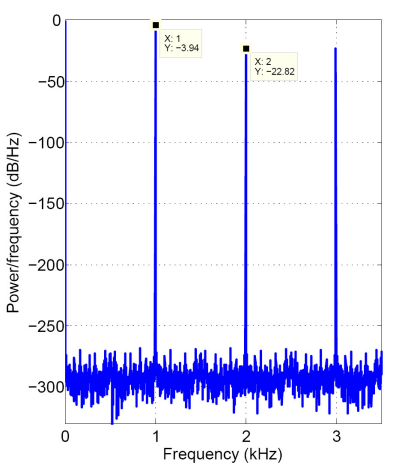

(a)

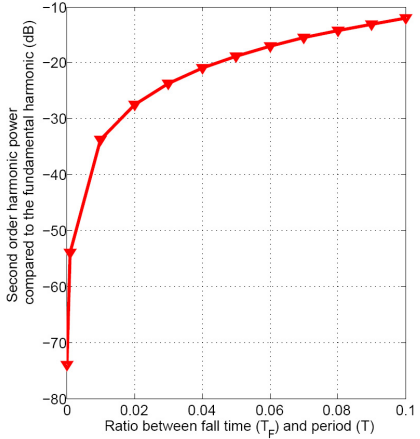

(b)
Figure 3. 3(a): Power spectral density of non-ideal sawtooth $\left(T_{F} / T=5 \%\right.$, $f_{s t}=1 \mathrm{kHz}$ ). Around $1 \mathrm{kHz}$ the power density is $-3.94 \mathrm{~dB} / \mathrm{Hz}$, and around $2 \mathrm{kHz}$ it is $-22.82 \mathrm{~dB} / \mathrm{Hz} ; 3(\mathrm{~b})$ : Second harmonic suppressions (compared to the fundamental harmonic) v.s. $T_{F} / T$.

peak-to-peak phase amplitude $2 n \pi(n \in \mathrm{N})$ and the repetition rate $f_{s t}$. The resultant frequency shift equals to $n f_{s t}$.

One important problem with the sawtooth phase modulation is that the fall-time of the phase $T_{F}$ can not be infinitely small (see figure 2), and that is due to the bandwidth limitation of the driver (function generator) and the modulator. In that case, the carrier and other harmonic sidebands are not totally suppressed [6]. As a result, the photocurrent signal from photodetector also has several unsuppressed sidebands with a spacing of $n f_{s t}$. Figure 3(a) illustrates the power spectral density of the photocurrent, when the reference signal is phase modulated to a non-ideal $1 \mathrm{kHz}$ sawtooth $\left(T_{F} / T=5 \%\right.$, where $T$ is the period of the sawtooth) and the measurement signal is kept unmodulated. The second-order harmonic is $19 \mathrm{~dB}$ weaker than the fundamental harmonic. The suppression of the second-order harmonic (compared to the fundamental harmonic) as a function of fall-time ratio is shown in figure 3(b).

\section{EXPERIMENT}

The heterodyne Mach-Zehnder interferometer (MZI) on SOI with grating coupler array is fabricated in IMEC, Leuven. (see figure 4). An optical fiber array with a fiber spacing of $250 \mu \mathrm{m}$ is used as a "bus" to send the laser into or out from the chip. The MZI has two SOI ridge waveguide arms (ridge: $220 \mathrm{~nm}$

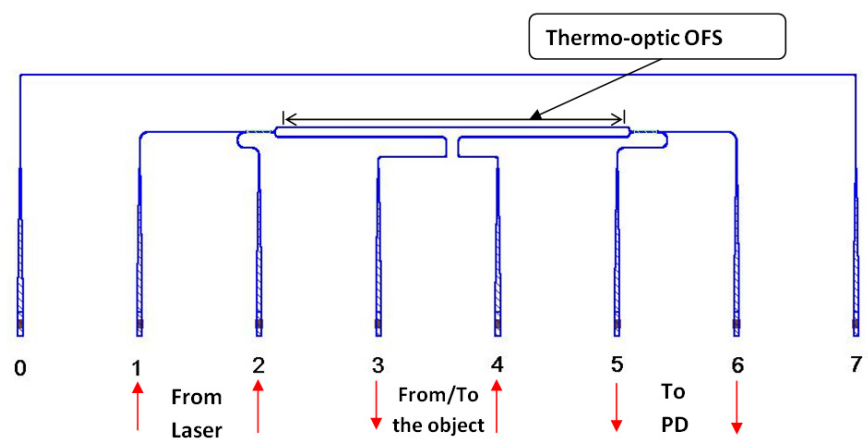

Figure 4. On-chip heterodyne MZI interferometer design.

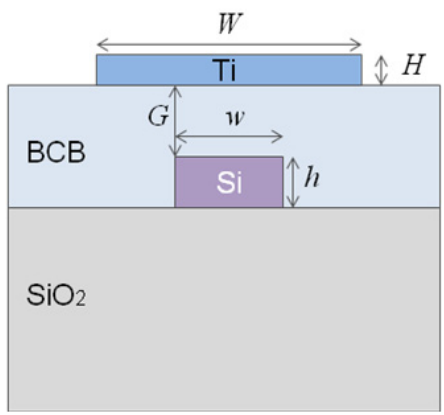

Figure 5. The cross-section of the thermo-optic frequency modulator.

high, $450 \mathrm{~nm}$ wide): the upper arm (725 $\mu \mathrm{m}$ long) used as the thermo-optic OFS, and the lower arm used for sending and receiving measurement light (via ports 3 and 4) to/from the moving object. A Ti heater will be fabricated on top of the upper waveguide arm, and is used to tune the effective index of the waveguide by changing the temperature. At both ends of the MZI, two 3dB MMIs are used as the optical splitter and combiner. A laser signal can be sent into the MZI via either port 1 or 2. Similarly, the photodetector can connect to either port 5 or 6 for optical receiving. The arm that links ports 0 and 7 is only used for the alignment between the fiber array and on-chip grating coupler array.

The sample is coated with a Benzocyclobutene (BCB) layer to separate the Ti heater from the waveguide, in order to avoid the large optical absorption caused by the metal. The thickness of the BCB between the top of the waveguide and the bottom of the heater $G$ is estimated to around $600 \mathrm{~nm}$. A $2 \mu \mathrm{m}$ width $(W), 320 \mathrm{~nm}$ thick $(H)$ Ti heater is then fabricated on top of the BCB by liftoff process. Measurements show that the resistance of this heater is $1.37 \mathrm{k} \Omega$. The cross-section of the modulator is shown in figure 5 .

While keeping port 3 and 4 connected with a fiber and a polarization controller (used for controlling the optical coupling efficiency between port 3 and 4), a $1550 \mathrm{~nm}$ laser is generated from a temperature controlled DFB laser and send to port 1 (or 2). An isolator and a polarization controller are used before the laser light enters to the chip. The isolator is employed to avoid the reflection of light to the DFB laser, and the polarization controller is used to couple the TE light into 


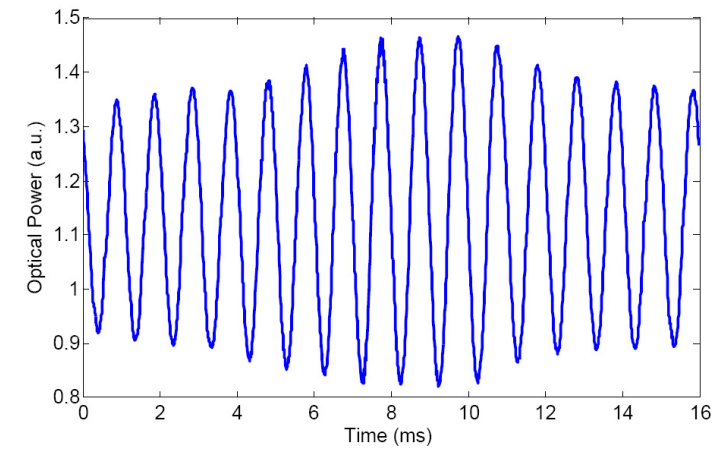

Figure 6. Optical power measured from the analog output of the powermeter as a function of time. An optimized thermo-optic serrodyne modulation (at $1 \mathrm{kHz}$ ) is applied to the reference arm. The measurement arm is connected with a fiber, but without any intended frequency shifted.

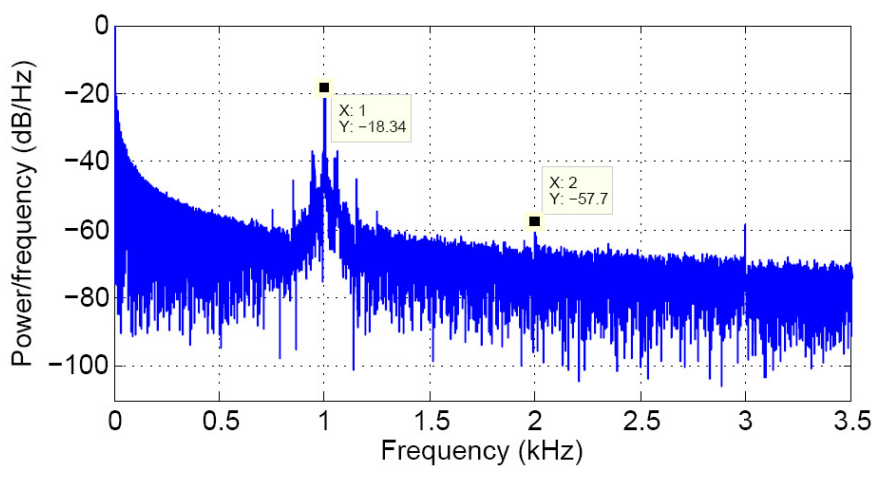

Figure 7. Power spectral density of the signal from the analog output. An optimized thermo-optic serrodyne modulation (at $1 \mathrm{kHz}$ ) is applied to the reference arm. The measurement arm is connected with a fiber, but without any intended frequency shifted.

the chip (through the grating couplers which are optimized for TE). At the output, a powermeter with an analog output is used to detect the time-varying optical power. The signal data is obtained by connecting the analog output of the powermeter to a data acquisition card (NI USB-6218) working at the sampling frequency of $100 \mathrm{ksps}$.

With a function generator, a controllable time-varying voltage is applied across the heater to heat the underlying waveguide and hence modulating its effective index. To generate a sawtooth temperature shape, the voltage should have a squareroot-of-time relation in each repetition period.

We generate a repetitive square-root-of-time voltage with a frequency of $1 \mathrm{kHz}$ across the heater. While keeping the lower level of the voltage zero, we change the higher voltage level to find the best value for the serrodyne frequency shift. It is found that, to suppress the second harmonic peak as much as possible, the peak-to-peak voltage across the heater has to be around $8.2 \mathrm{~V}$ (corresponds to a thermal power of $46 \mathrm{~mW}$ ). The optical power from the analog output in such situation is shown in figure 6 . The power spectral density of the analog output signal is shown in figure 7.

From figure 7, it is seen that the second-order harmonic is $39 \mathrm{~dB}$ weaker than the fundamental harmonic frequency.
Assume that the fall-time (limited by thermal time constants) is the main cause of the higher order harmonics, this reveals that the fall-time is less than $20 \mu$ s according to $3(\mathrm{~b})$. It is also found that there are several small sidebands around the fundamental harmonic peak. They are about $50 \mathrm{~Hz}$ apart from each other and are most probably caused by vibrations of the fiber connecting to ports 3 and 4 .

\section{CONCLUSION}

It is reported that the $1 \mathrm{kHz}$ optical frequency shift on SOI using the thermo-optic serrodyne modulation has been generated. The driving voltage across the $\mathrm{Ti}$ heater has a square-root-of-time shape in each period, and is $8.2 \mathrm{~V}$ peak-topeak. The average power of the OFS is $23 \mathrm{~mW}$. The secondorder harmonic peak suppression is $-39 \mathrm{~dB}$ relative to the fundamental harmonic.

\section{ACKNOWLEDGMENT}

The authors would like to thank Gunay Yurtsever, Jonathan Schrauwen Steven Verstuyft, Shahram Keyvaninia, Stevan Stankovic, Katarzyna Komorowska, and Zon-Qiang Yu for their help. We would also like to acknowledge ePIXfab for the fabrication of SOI chips.

\section{REFERENCES}

[1] "Instability and unsteadiness of aircraft wake vortices," Aerospace Science and Technology, vol. 7, no. 8, pp. 577 - 593, 2003.

[2] G. Bissinger and D. Oliver, "3-D laser vibrometry on legendary old Italian violins," Sound And Vibration, vol. 41, no. 7, pp. 10-15, 2007.

[3] T. Tanaka, C. Riva, and I. Ben-Sira, "Blood Velocity Measurements in Human Retinal Vessels," Science, vol. 186, no. 4166, pp. 830-831, 1974.

[4] G. S. Martin Johansmann and M. Pineda, "Targeting the limits of laser doppler vibrometry," Polytec, Tech. Rep., Octoer 2005.

[5] A. Waz, P. Kaczmarek, and K. Abramski, "Laser-fibre vibrometry at $1550 \mathrm{~nm}, "$ Measurement Science \& Technology, vol. 20, no. 10, p. 105301 (8 pp.), October 2009.

[6] L. Johnson and C. Cox, "Serrodyne optical frequency translation with high sideband suppression," Journal Of Lightwave Technology, vol. 6, no. 1, pp. 109-112, Jan 1988.

[7] M. W. Pruessner, T. H. Stievater, M. S. Ferraro, and W. S. Rabinovich, "Thermo-optic tuning and switching in SOI waveguide Fabry-Perot microcavities," Optics Express, vol. 15, no. 12, pp. 7557-7563, JUN 112007.

[8] William M. Green and Michael J. Rooks and Lidija Sekaric and Yurii A. Vlasov, "Ultra-compact, low RF power, $10 \mathrm{~Gb} / \mathrm{s}$ silicon Mach-Zehnder modulator," Opt. Express, vol. 15, no. 25, pp. 17 106-17 113, 2007.

[9] M. B. Duhring and O. Sigmund, "Improving the acousto-optical interaction in a Mach-Zehnder interferometer," Journal Of Applied Physics, vol. 105, no. 8, Apr 152009.

[10] P. Dumon, W. Bogaerts, A. Tchelnokov, J.-M. Fedeli, and R. Baets, "Silicon nanophotonics," Future Fab International (invited), vol. 25, pp. 29-36, 2008. 